\title{
Editorial
}

\section{Nutrition and Cancer}

Nowadays, cancer is recognized to be not only one disease but an expression of multiple and unique aberrations of the normal life processes of cells (e.g., growth, differentiation and death). Nutrition has been suspected to play an important role in cancer etiology, incidence and prognosis. Furthermore, it is thought to be the most influential of all external environmental factors due to its ability to affect the transcriptional activity and expression of certain genes. However, the knowledge of the roles of nutrition which are protective or contribute to carcinogenesis is limited or equivocal.

A healthy lifestyle, including healthy eating (eating more fruit, vegetables and whole grain foods), regular exercise, avoiding overweight, not smoking and limiting the intake of alcohol are recommended as helping to reduce the risk of cancer. Nevertheless, until now no dietary changes can be guaranteed to prevent cancer. Early epidemiologic cancer studies seemed to confirm the link between nutrition and cancer. However, large, prospective cohorts do not support such a link. Given the difficulties in precisely assessing habitual diet, the potential importance of nutrition during the life span remains to be explored.

Understanding the role of nutrition in the cancer process requires a more comprehensive and thorough understanding of the regulations that occur within the genetic, epigenetic, proteomic and metabolic domains. Although the challenges to understanding these relationships are enormous, the societal benefits are indubitable.

In this issue of Gastrointestinal Tumors, the selected aspects of nutrition and cancer will provide a detailed summary of and excellent update on this interesting topic. It is my hope that everyone gains useful information with regard to understanding the potential link between nutrition and cancer after having read this special issue.

Prof. Jing-Yuan Fang, Shanghai 